Proyecciones

Vol. $13 N^{\circ} 1$, pp.01-07 July 1994

Universidad Católica del Norte

Antofagasta - Chile

\title{
BLOCK DIAGONALIZATION OF SYSTEMS WITH MEASURABLE COEFFICIENTS*
}

\author{
RAÚL NAULIN
}

I.M.A Universidad Católica de Valparaíso, Valparaiso, Chile.

\begin{abstract}
In this paper we show that, previous results given by Coppel concerning the existence of projection matrix $P$, and a change of variable $x=S(t) y$ reducing system $\dot{x}=A(t) x$, where $A(t)$ is a continuous matrix function, to the form $\dot{y}=\hat{A}(t) y$, with the property $P \hat{A}(t)=\hat{A}(t) P$, can be extended to the case when $A(t)$ is a locally integrable function.
\end{abstract}

Key Words and Phrases: Diagonalization of linear systems, systems with Carathéodory conditions.

A.M.S. Classification: $34 A 05,34 E 05$ 


\section{INTRODUCTION}

The methods of the theory of dichotomies has been recognized as a an important tool in the application and theoretical research of ordinary differential equations [6], impulsive differential equations [2, 10], functional differential equations [4] and difference equations [1]. The notion of exponential dichotomy for non autonomous systems corresponds to the notion of hyperbolicity of autonomous system. One can say that the theory of exponential dichotomies establishes a bridge beetween the geometrical methods of dynamical systems and the theory of non autonomous systems [11]. This work concerns with an application of this theory to the problem of block diagonalization of systems with discontinuous coefficients.

In this work we consider the linear system

$$
\dot{x}=A(t) x, \quad x \in \mathbf{R}^{n}
$$

where $A(t)$ is a matrix value function defined on some interval $J$. We assume that (1) is a Caratheodory system [3], that is $A(t)$ is integrable on any compact interval.

We investigate the following problem: given $P$, a projection matrix, we look for a transformation $x(t)=T(t) y(t)$ such that transformed system has the following properties:

$$
\begin{aligned}
y^{\prime} & =\hat{A}(t) y \\
\hat{A}(t) & :=T^{-1}(t) A(t) T(t)-T^{-1}(t) \dot{T}(t) \\
P \hat{A}(t) & =\hat{A}(t) P
\end{aligned}
$$

In virtue of that any projection $P$ is similar to a projection of the form $\left(\begin{array}{cc}I_{k} & 0 \\ 0 & 0\end{array}\right)$, we have that the system (2) with the property (3) can be written in appropiate coordinates as a decoupled system

$$
\begin{gathered}
\dot{u}=\hat{A}_{1}(t) u \\
\dot{v}=\hat{A}_{2}(t) v \\
\hat{A}(t)=\operatorname{diag}\left(\hat{A}_{1}(t), \hat{A}_{2}(t)\right) .
\end{gathered}
$$

This important problem has been studied by Coppel in $[5,6]$, where the following result is proved:

Theorem 1. Suppose that the function $A(t)$ is continuous and for a fundamental matrix $\Phi(t)$ of (1) and an orthogonal projection $P$ we have that $\Phi(t) P \Phi^{-1}(t)$ is bounded on $J$, then there exists a change of variable $x(t)=T(t) y(t), T(t)$ and $T^{-1}(t)$ are bounded functions, reducing (1) to the system (2) with the property (3). 
In the theorem above the conditions of continuity imposed on $A(t)$ restricts the application of this result to a wide class of systems, namely systems with Carathéory conditions $[8,3]$. In [6] Coppel does not emphasize the fact that his results can be extended to this class of systems. In this article we show that with appropiate modifications Coppel's results produces the following

Theorem 2. If $A(t)$ is a locally integrable functions, and for some fundamental matrix $X(t)$ and some orthogonal projection $P$ we have that $X(t) P X^{-1}(t)$ is bounded, then there exists an absolutely continuous function

$$
S(t): J \longrightarrow \mathrm{C}^{n \times n},
$$

such that the change of variable $x=S(t) z$ reduces the system (1) to

$$
z^{\prime}=B(t) z
$$

with the properties

$$
\begin{aligned}
|B(t)| & \leq \sqrt{n}|A(t)| \\
P B(t) & =B(t) P, \forall t \in J
\end{aligned}
$$

Moreover, the functions $S(t)$ and $S^{-1}(t)$ are bounded.

2. LEMMATA ON SQUARE ROOTS OF POSITIVE MATRICES

Let $\left(\mathcal{M}_{n}, \mathbf{R}\right)$ denote the vector space, over $\mathbf{R}$, of hermitian, $n \times n$ matrices with complex coefficients. We say that a matrix $A \in \mathcal{M}_{n}$ is positive (we write $A>0$ ) if for any $\xi \in \mathbf{C}^{n}, \xi \neq 0$, we have $\left.(\xi, A \xi)>0,(<u, v\rangle=\sum u_{i} \bar{v}_{i}\right)$. Clearly $\left(\mathcal{M}_{n}, \mathbf{R},||\right)$ is a Banach space. Let $\mathcal{P}_{n}$ denote the set of positive matrices in $\mathcal{M}_{n}$.

The following lemma is a well known fact of analysis [7].

Lemma 1. The set $\mathcal{P}$ is open in $\left(\mathcal{M}_{n}, \mathbf{R}\right)$.

From the implicit function theorem [11] is obtained the proof of the following

Lemma 2. Let $A_{0} \in \mathcal{P}$, then there exists a neighborhood of $A_{0}, U\left(A_{0}\right)$ such that for any $A \in U\left(A_{0}\right)$ there exists a unique $B \in \mathcal{P}$ such that $B^{2}=A$. Moreover the application $\phi: U\left(A_{0}\right) \longrightarrow \mathcal{P}$ defined as $\phi(A)=B$, where $B^{2}=A$, is infinitely differentiable.

We will denote $\phi(A)=\sqrt{A}$, for $A \in U\left(A_{0}\right)$.

Theorem 3. Let $T(t):[a, b] \longrightarrow \mathcal{P}$ be a absolutely continuous function, then $\sqrt{T(t)}$ is absolutely continuous. 
Proof: $\quad$ For any $t^{*} \in[a, b]$ we can construct a ball $B\left(T\left(t^{*}\right), \delta_{t} \cdot\right)$, and a function $\phi_{t^{*}}: \quad B\left(T\left(t^{*}\right), \delta_{t^{*}}\right) \longrightarrow \mathcal{P}$ such that $\sqrt{A}=\phi_{t} \cdot(A)$, for all $A \in$ $B\left(T\left(t^{*}\right), \delta_{t^{*}}\right)$. The conectedness and compactness of the set $\{T(t), t \in[a, b]\}$ permits to choise a finite number of balls $B\left(T\left(t_{k}\right), \delta_{k}\right), k=1,2, \ldots, m$, which cover $\{T(t), t \in[a, b]\}$ and a function

$$
\phi: \bigcup_{k=1}^{m} B\left(T\left(t_{k}\right), \delta_{k}\right) \longrightarrow \mathcal{P}
$$

with the property $A=\phi^{2}(A)$. $\phi$ is differentiable with bounded derivatives. In this manner the composition $\phi(T(t))$ is an absolutely continuous function.

Lemma 3. Let $P$ be an orthogonal projection (that is $P^{2}=P$ and $P=P^{*}$ ) and $P T(t)=T(t) P$, then $P \sqrt{T(t)}=\sqrt{T(t)} P$.

Proof: If $A$ is a positive matrix, $P A=A P$ and $P^{*}=P$ then $P \sqrt{A}=\sqrt{A} P$. This fact is proven in [7].

\section{Projection MATRIX FUNCTIONS}

Let $X: J \longrightarrow \mathrm{C}^{n \times n}$ be a matrix function such that $X(t)$ is invertible for any $t \in J$. Let $P$ be a projection, then the function $P(t):=X(t) P X^{-1}(t)$ satisfies $P^{2}(t)=P(t)$ for all $t \in J$. In [3] Coppel proves the following remarkable fact: if $X(t) \in C^{1}$, then there exists a function $S: J \longrightarrow \mathrm{C}^{n \times n} S(t) \in C^{1}$ and $S(t)$ is bounded, such that $P(t)=S(t) P S^{-1}(t)$. It turns out that this result can be extended for absolutely continuous functions $X(t)$ :

Theorem 4. Let $P$ be an orthogonal projection and

$$
X(t): J \longrightarrow \mathrm{C}^{n \times n}
$$

be an absolutely continuous function which is invertible for any $t \in J$. Then there exists a bounded continuous function

$$
S: J \longrightarrow \mathbf{C}^{n \times n}
$$

invertible for any $t \in J$, such that:

(i) $S P S^{-1}=X P X^{-1}$

(ii) $|S| \leq \sqrt{2}$

(iii) $\left|S^{-1}\right| \leq\left\{\left|X P X^{-1}\right|^{2}+\left|X(I-P) X^{-1}\right|^{2}\right\}^{1 / 2}$

(iv) $S(t)$ is absolutely continuous 
Proof: Let

$$
R(t):=\sqrt{P X^{*} X P+(I-P) X^{*} X(I-P)}
$$

then $R(t)$ is positive for any $t$, and by the Lemma $3, P R(t)=R(t) P$. According to Theorem 3, $R(t)$ is absolutely continuous. We define $S(t)=X(t) R^{-1}(t)$. Then $\mathrm{S}(\mathrm{t})$ is absolutely continuous, since it is defined as the product of two absolutely continuous functions. The rest of the proof is identical to that given in Lecture 5 , Lemma 1 in $[6]$.

\section{Block diagonalization of linear Carathéodory systems}

In what follows, we will denote throughout $P$ an orthogonal projection matrix. By $X(t)$ will we denote a fundamental matrix of system (1).

Theorem 5. The change of variable $z=S(t) x$, where $S(t)$ is defined in Theorem 4 reduces (1) to the form

$$
\dot{z}=B(t) z, B(t)=S^{-1}\left(A S-S^{\prime}\right)(t)
$$

with the property $P B(t)=B(t) P$ for $t \in J$. If $X\left(t^{*}\right)=I$ for some $t^{*} \in J$, then $S\left(t^{*}\right)=I$ and $R\left(t^{*}\right)=I$.

Proof: First we note that

$$
\begin{aligned}
B & =S^{-1}\left(A S-S^{\prime}\right)=S^{-1} A S-S^{-1} S^{\prime} \\
& =S^{-1} A S-S^{-1}\left(X^{\prime} R^{-1}-X R^{-1} R^{\prime} R^{-1}\right) \\
& =S^{-1} A S-S^{-1} A S+S^{-1} X R^{-1} R^{\prime} R^{-1}=R^{\prime} R^{-1}
\end{aligned}
$$

This means that the function $B(t)$ is measurable and $R(t)$ is a fundamental matrix of $(3)$.

From Lemma 3 we have $P R(t)=R(t) P$. Therefore $P R^{\prime}=R^{\prime} P=B(t) P R=$ $P B(t) R$ for any $t \in J$.

The following Theorem 6 will proves that (4) is a Carathéodory system, previously we need the following:

Lemma 4. Let $\phi: \mathcal{P} \longrightarrow \mathcal{P}$ is a function defined by the equation $A=\phi^{2}(A), \forall A \in$ $\mathcal{P}$, then $\phi \in C^{\infty}$ and

$$
\phi^{\prime}(A) B=\int_{0}^{\infty} e^{-\phi(A) s} B e^{-\phi(A) s} d s
$$

Proof: We begin by observing that for a positive matrix $A$ and any square $n \times n$ matrix $B$, the algebraic system

$$
U \phi(A)+\phi(A) U=B
$$


has a unique solution, namely:

$$
U=\int_{0}^{\infty} e^{-\phi(A) t} B e^{-\phi(A) t} d t
$$

From Lemma 2, we know that the function $\phi(A)=\sqrt{A} \in C^{\infty}$. We will calculate the derivative of $\phi$ at any point $A$. Since

$$
\phi(A) \phi(A)=A, \phi(A+h) \phi(A+h)=A+h,
$$

then

$$
\phi(A+h)=\phi(A)+\phi^{\prime}(A) h+o(h)
$$

and

$$
\left(\phi(A)+\phi^{\prime}(A) h+o(h)\right)\left(\phi(A)+\phi^{\prime}(A) h+o(h)\right)=A+h,
$$

This last identity can be written in the form:

$$
A+\phi^{\prime}(A) h \phi(A)+\phi(A) \phi^{\prime}(A) h+\phi^{\prime}(A) h \phi^{\prime}(A) h=A+h+o(h) .
$$

If $h=t B$, with $t \in \mathbf{R}, t>0$, then:

$$
t \phi^{\prime}(A) B \phi(A)+t \phi(A) \phi^{\prime}(A) B=t B+o(t B)
$$

and

$$
\phi^{\prime}(A) B \phi(A)+\phi(A) \phi^{\prime}(A) B=B+o(t B) / t .
$$

Letting $t \rightarrow 0$, we obtain from (4) and (5):

$$
\phi^{\prime}(A) B=\int_{0}^{\infty} e^{-\phi(A) t} B e^{-\phi(A) t} d t
$$

Formula (6) allows to estimate the norm of the derivative $R /(t)$ and this is all we need to extend the proof of Theorem 6, whose proof belongs to Coppel [5], to systems with locally integrable coefficients.

Theorem 6. $|B(t)| \leq \sqrt{n}|A(t)|$ almost everywhere on $J$.

Proof of Theorem 2: $\quad$ The function $\mathrm{S}(\mathrm{t})$ giving the change of variable $X=$ $S(t) y$ is defined in Theorem 4 with the bound $|S(t)| \leq \sqrt{2}$; From the boundedness of

$$
\left|X(t) P X^{-1}(t)\right| \leq M
$$

and Theorem 4 (ii) we obtain the boundedness of $S^{-1}(t)$. Theorem 4 (iv) says that $\mathrm{S}(\mathrm{t})$ is absolutely continuous. From Theorem 5 we have the conmutativity $P B(t)=B(t) P$ in system (4). Finally Theorem 6 states the local integrability of function $B(t)$. 


\section{References}

[1] Agarwal, R.P., Difference Equations and Inequalities, Pure and Aplied Mathematics, Marcel Dekker, New York, 1992.

[2] Bainov, D.D., Simeonov, P.S., Systems with Impulse Effect, Ellis Horwood and John Wiley, New York, 1989

[3] Coddington E., Levinson N., Theory of Ordinary Differential Equations, Mc Graw Hill, New York, 1955.

[4] Cooke, K., Asymptotic theory for the delay differential equation, $u \prime=-a u(t-$ $r(t))$, J. Math. An. and App., Vol. 19, 1, pp. 160-173, 1967.

[5] Coppel W.A., Dichotomies and reducibility, Journal of differential equations $3,500-521,(1967)$.

[6] Coppel W.A., Dichotomies in Stability Theory, Lectures Notes in Mathematics, 629, Springer-Verlag, Berlin, 1978.

[7] Kreyszing E., Introductory Functional Analysis with Applications, John Wiley, New York, 1978.

[8] Lee E., Markus L., Foundations of Optimal Control Theory, John Wiley and Sons, New York, 1967.

[9] Naulin R., Dichotomies for systems with unbounded coefficients, IMA, UCV, Valparaíso, 1992 (Preprint).

[10] Naulin, R., Pinto, M., Block diagonalization of linear impulsive systems, Facultad de Ciencias, Universidad de Chile, 1992(preprint).

[11] Palmer, K., Exponential dichotomies and transversal homoclinic points, J. Diff. Eqns., 55, pp. 225-256, 1984.

[12] Prodi G., Ambrosetti A., Analisi non Lineare, I Quaderno, Scuola Normale Superiore, Pisa, 1973.

Received: December 12, 1992

Raúl Naulin

Instituto de Matemáticas

Universidad Católica de Valparaiso

Casilla 4059, Valparaiso, Chile 